\title{
Enhanced ductility of nanomaterials through cooperative dislocation emission from cracks and grain boundaries
}

\author{
Hui Feng ${ }^{\mathrm{a}}$, Jianming Pang ${ }^{\mathrm{a}}$, Qihong Fang ${ }^{\mathrm{a}^{*}}$, Changping Chen ${ }^{\mathrm{b}}$, Pihua Wen ${ }^{\mathrm{c}}$ \\ ${ }^{a}$ State Key Laboratory of Advanced Design and Manufacturing for Vehicle Body, Hunan University, Changsha \\ 410082, PR China \\ ${ }^{\mathrm{b}}$ School of Civil Engineering and Architecture, Xiamen University of Technology, Xiamen, 361024, PR China \\ ${ }^{\mathrm{c}}$ School of Engineering and Material Sciences, Queen Mary, University of London, London E14NS, UK
}

\begin{abstract}
An analytical model is established to explore the cooperative mechanism between the dislocation emission from cracks and grain boundaries driven by grain boundary sliding in deformed nanocrystalline materials. In our model, high local stress concentration nearby the crack actives grain boundary sliding which creates a wedge disclination dipole at the grain boundaries' triple junctions. The grain size-dependent criterions for the dislocation emission from the crack tip and the grain boundary are respectively derived. Influences of grain boundary sliding and grain size on the cooperative mechanism are discussed. The results show that the dislocation emission from the grain boundary is activated ahead of that from the crack tip for small grain sizes. This can explain that grain boundary sliding can toughen the nanocrystalline materials even though it suppresses dislocation emission from cracks when their grain sizes are relative small, which is because the dislocation emission from grain boundaries is activated. With the increasing grain size, the main dislocation source may transform from grain boundaries to crack tips due to grain boundary sliding. Therefore, the ductility of nanomaterials with different grain sizes can be enhanced through the cooperative dislocation emission from cracks and grain boundaries.
\end{abstract}

Keywords: Nanocrystalline material; Cooperative mechanism; Dislocation emission; Crack tip; Grain boundary.

\footnotetext{
${ }^{*}$ Corresponding author:

Email: fangqh1327@hnu.edu.cn (Q.H. Fang)
} 


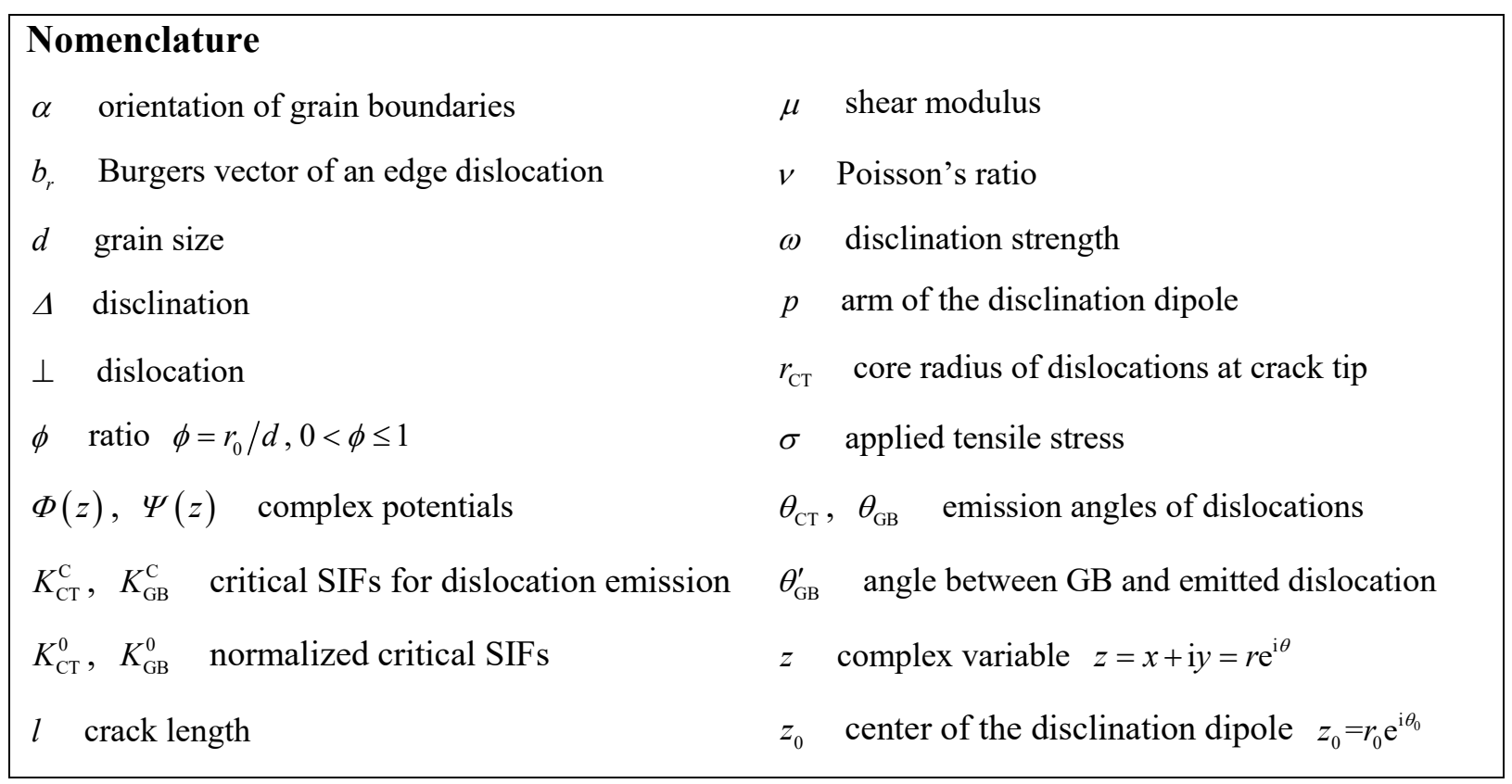

\section{Introduction}

Nanocrystalline (NC) metals and ceramics have exhibited remarkable mechanical properties, e.g., high strength and hardness, indicating a great capacity for many applications [1-10]. However, in most cases, NC materials show disappointing fracture toughness and low tensile ductility at room temperatures, which considerably restricts their practical utility [1-3]. Nevertheless, certain experimental results demonstrate that NC materials can exhibit the combination of superior strength and good tensile ductility [11-15]. The reason why NC materials have so unique peculiarities is that NC materials are characterized by the specific structural features compared to coarse-grained materials. For instance, nanoscale grains and high density of grain boundaries (GBs) play a major role in their fracture behaviors. On one hand, as a result of high density GBs, specific deformation modes are proposed, e.g., GB sliding and migration, Coble creep, nanoscale amorphization and deformation twinning [6, 16-22]. These specific deformation mechanisms can not only release the high-concentrated stress, which will effectively impede the initiation of nanoscale cracks, but also retard or even prevent the propagation of formed cracks. 
On the other hand, with the decreasing of grain size down to nanoscale, the conventional lattice dislocation slip, which is the main plastic deformation mode for coarse-grained metals, is hindered or even completely suppressed due to the arrest effect of the high density of GBs [2]. The size effect of nanoscale grains prevents the activity of lattice dislocation sources, e.g., Frank-Read sources [23]. Nevertheless, dislocations can generate at crack surfaces and lattice dislocation emission from cracks will contribute to the plastic deformation of NC materials [24-29]. The "in-situ" observation (by high-resolution electron microscopy) of edge dislocation emission from a crack tip during the deformation of NC Ni has been reported by Kumar et al. [30]. Besides, the internal back stress produced by the emitted dislocations can accommodate the stress intensity at the crack tip area due to the applied load, which causes effective crack blunting, therefore prevents crack propagation and strengthens the fracture toughness.

Meanwhile, GBs also serve as sources of the lattice dislocations [7, 31]. For instance, GB dislocations accumulated at triple junctions can be generated by intergrain sliding [32, 33]. High stresses also can activate the dislocation emission from GBs along the direction approximately normal to the GB plane [34, 35]. Recently, Ovid'ko and Skiba [36] suggested a novel mechanism describing the emission of partial dislocations from GBs driven by intergrain sliding in NC materials. The enhanced dislocation emission can effectively release the stress concentration, which competes against the nucleation of nanocrack. The ductility of $\mathrm{NC}$ materials will be improved if the dislocation emission from GBs can well cooperate with intergrain sliding.

Both dislocation emission from the cracks and GBs play important roles in the toughening of NC materials. While which dislocation emission mechanism will be first activated during the deformation? Which one plays the dominant role? Hitherto, it has not been reported yet. 
In this article, we study the cooperative mechanism between dislocation emission from a crack and GB driven by GB sliding in a deformed NC solid. GB sliding, which is a specific plastic deformation mode contributing to the toughening of NC materials, can be activated under the high concentrated stresses operating in the vicinity of the crack tip. What's more, GB dislocations and dipole of disclinations can be generated during the GB sliding due to the deformation incompatibilities [37-40]. In the meantime, GB sliding can serve as stress sources providing the dislocation emission from the GB [36] and influence the dislocation emission from the crack tip [38]. The grain size-dependent criterions for the dislocation emission from the crack tip and GB are derived, respectively. Then, the effect of GB sliding and grain size on the dislocation emission is discussed in detail.

\section{Modeling}

Consider a deformed isotropic NC specimen with the shear modulus $\mu$ and the Poisson's ratio $v$, in which a flat crack of length $l$ is formed under the action of the applied tensile stress $\sigma$. The crack is assumed to propagate perpendicularly to the direction of the applied tensile stress (Fig. 1). For simplicity, the defect structure of the solid is assumed to be the same along the coordinate axis $z$ perpendicular to the xoy-plane. This assumption will allow us to restrict our consideration to a two-dimensional grain structure which definitely reflects the key aspects of the problem.

High concentrated stresses nearby the crack tip can activate high-angle GB sliding nearby the crack tip. Following the theory of defects, wedge disclination dipoles can be generated at the GB junctions during the process of GB sliding $[39,40]$. In the paper, the situation where GB sliding comes up at the closest junction $B$ will be considered, as shown in Fig.1. The wedge disclination dipole at $B\left(z_{1}=z_{0}-\mathrm{ie}^{\mathrm{i} \alpha} p / 2\right)$ and $B^{\prime}\left(z_{2}=z_{0}+\mathrm{ie}^{\mathrm{i} \alpha} p / 2\right)$ characterized by strength $\pm \omega$ can be formed due to $B B^{\prime}$ GB sliding [20]. The center of the disclination 
dipole $B B^{\prime}$ locates at the point $z_{0}\left(=r_{0} \mathrm{e}^{\mathrm{i} \theta_{0}}\right)$ and its arm is represented as $p . \alpha$ denotes the GB's orientation.

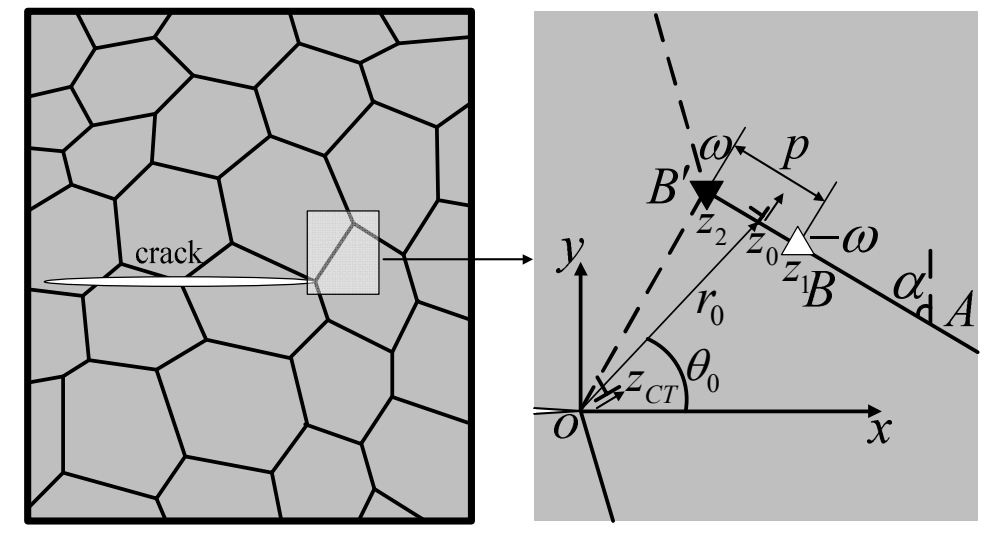

(a)

(b)

Figure 1. (a) A deformed NC specimen with a mode I crack under the applied tensile stress; (b) GB sliding nearby a crack tip and dislocations emitting from the crack tip and the GB.

First of all, the stress field produced by the GB sliding (a dipole of wedge disclinations) nearby the crack tip should be derived. Utilizing the Muskhelishvili's complex variable method, two complex potentials $\Phi(z)$ and $\Psi(z)$ are introduced to express the stress field [41]:

$$
\begin{gathered}
\sigma_{x}=\operatorname{Re}\left[2 \Phi(z)-\bar{z} \Phi^{\prime}(z)-\Psi(z)\right], \\
\sigma_{y}=\operatorname{Re}\left[2 \Phi(z)+\bar{z} \Phi^{\prime}(z)+\Psi(z)\right], \\
\tau_{x y}=\operatorname{Im}\left[\bar{z} \Phi^{\prime}(z)+\Psi(z)\right],
\end{gathered}
$$

with $z=x+\mathrm{i} y, \Phi^{\prime}(z)=\mathrm{d}[\Phi(z)] / \mathrm{d} z$ and the over-bar denotes the conjugate of complex.

For the present issue, $\Phi_{\Delta}(z)$ and $\Psi_{\Delta}(z)$ (the subscript ' $\Delta$ ' represents the disclination) will be expressed as:

$$
\begin{aligned}
& \Phi_{\Delta}(z)=\Phi_{\Delta 0}(z)+\Phi_{\Delta}^{*}(z), \\
& \Psi_{\Delta}(z)=\Psi_{\Delta 0}(z)+\Psi_{\Delta}^{*}(z),
\end{aligned}
$$


where $\Phi_{\Delta 0}(z)$ and $\Psi_{\Delta 0}(z)$ denote the complex potentials for a dipole of wedge disclination in an infinite homogeneous solid. $\Phi_{\Delta}^{*}(z)$ and $\Psi_{\Delta}^{*}(z)$ represent the disturbance of the complex potentials due to the interaction with the crack, which are both holomorphic.

For simplicity, the crack is assumed to lie on the $x$-axis. According to the refferences [38, 42, 43], $\Phi_{\Delta}(z)$ and $\Psi_{\Delta}(z)$ associated with the wedge disclinations respectively locating at points $z_{1}$ and $z_{2}$ can be given as:

$$
\begin{gathered}
\Phi_{\Delta}(z)=\frac{\mu \omega}{8 \pi(1-v)} \sum_{k=1}^{2}(-1)^{k}\left(\ln \frac{z-z_{k}}{z-\bar{z}_{k}}-\frac{z-z_{k}}{z-\bar{z}_{k}}\right) \\
+\frac{\mu \omega}{8 \pi(1-v)} X_{0}(z) \sum_{k=1}^{2}(-1)^{k}\left(\frac{1}{X_{0}\left(z_{k}\right)} \ln \left(z-z_{k}\right)-z_{k}+\bar{z}_{k}\right. \\
\left.-\frac{1}{X_{0}\left(\bar{z}_{k}\right)}\left(\frac{z-z_{k}}{z-\bar{z}_{k}}+\ln \left(z-\bar{z}_{k}\right)\right)\right), \\
\Psi_{\Delta}(z)=\frac{\mu \omega}{8 \pi(1-v)}\left(\sum_{k=1}^{2}(-1)^{k}\left(\frac{3 z-z_{k}}{z-\bar{z}_{k}}-\frac{\bar{z}_{k}}{z-z_{k}}-\frac{z\left(z-z_{k}\right)}{\left(z-\bar{z}_{k}\right)^{2}}\right)\right) \\
-\frac{\mu \omega}{8 \pi(1-v)} X_{0}(z) \sum_{k=1}^{2}(-1)^{k}\left(\frac{1}{X_{0}\left(z_{k}\right)} \frac{\bar{z}_{k}}{z-z_{k}}+\frac{1}{X_{0}\left(\bar{z}_{k}\right)}\left(\frac{z_{k}-3 z}{z-\bar{z}_{k}}+\frac{z\left(z-z_{k}\right)}{\left(z-\bar{z}_{k}\right)^{2}}\right)\right) \\
-\frac{\mu \omega}{8 \pi(1-v)} X_{0}^{\prime}(z) \sum_{k=1}^{2}(-1)^{k}\left(\frac{z}{X_{0}\left(z_{k}\right)} \ln \left(z-z_{k}\right)-z\left(z_{k}-\bar{z}_{k}\right)\right. \\
-\frac{z}{X_{0}\left(\bar{z}_{k}\right)}\left(\frac{z-z_{k}}{z-\bar{z}_{k}}+\ln \left(z-\bar{z}_{k}\right)\right),
\end{gathered}
$$

with $X_{0}(z)=1 / \sqrt{z(z-l)}$ and $X_{0}^{\prime}(z)=\mathrm{d}\left[X_{0}(z)\right] / \mathrm{d} z$.

With the substitution of Eqs. (6) and (7) into formula (1)-(3), it is easy to derive the stress field $\left(\sigma_{x}, \sigma_{y}\right.$ and $\left.\tau_{x y}\right)$ produced by the interaction between the GB sliding and the flat crack.

\section{Emission force of the dislocations}

Considering the influence of GB sliding, the dislocations emitting from the crack tip and GB are respectively studied. To compare the two types of dislocation emission, it is necessary 
to figure out the force acting on the dislocations first. For simplicity, we focus on the situation where the dislocations are of edge character.

\subsection{Dislocation emission from crack tip}

For the first dislocaiton emitting from the crack tip, the emission force $f_{\text {emit }}^{\mathrm{CT}}$ contains three parts: the self-image force $f_{\mathrm{I}}^{\mathrm{CT}}$, the force $f_{\Delta}^{\mathrm{CT}}$ induced by the GB sliding and the external force $f_{\mathrm{A}}^{\mathrm{CT}}$.

Firstly, for an edge dislocation with Burgers vector $b_{r} \mathrm{e}^{\mathrm{i} \theta_{\mathrm{e}}}$ locating at $z_{\mathrm{CT}}=r_{\mathrm{CT}} \mathrm{e}^{\mathrm{i} \theta_{\mathrm{CT}}}$, the elastic stress field can be derived using the Muskhelishvili's complex variable method. Introducing $\Phi_{\perp}(z), \Psi_{\perp}(z)$ and $\Omega_{\perp}(z)$ and following Fang et al. [44], $\Phi_{\perp}(z)$ and $\Psi_{\perp}(z)$ are given as:

$$
\begin{aligned}
& \Phi_{\perp}(z)=\Phi_{\perp 0}(z)+\Phi_{\perp}^{*}(z), \\
& \Psi_{\perp}(z)=\Psi_{\perp 0}(z)+\Psi_{\perp}^{*}(z),
\end{aligned}
$$

with $\Phi_{\perp 0}(z)=w /\left(z-z_{\mathrm{CT}}\right), \quad \Psi_{\perp 0}(z)=\bar{w} /\left(z-z_{\mathrm{CT}}\right)+w \bar{z}_{\mathrm{CT}} /\left(z-z_{\mathrm{CT}}\right)^{2}$, and $w=\mu b_{r}\left(\sin \theta_{\mathrm{e}}-\mathrm{i} \cos \theta_{\mathrm{e}}\right) / 4 \pi(1-v)$.

By the same method used to derive the complex potentials $\Phi_{\Delta}(z)$ and $\Psi_{\Delta}(\mathrm{z})$, we can obtain:

$$
\begin{aligned}
\Phi_{\perp}(z)= & \frac{1}{2}\left(\frac{w}{z-z_{\mathrm{CT}}}-\frac{w}{z-\bar{z}_{\mathrm{CT}}}-\frac{\bar{w}\left(z_{\mathrm{CT}}-\bar{z}_{\mathrm{CT}}\right)}{\left(z-\bar{z}_{\mathrm{CT}}\right)^{2}}\right) \\
& +\frac{1}{2} X_{0}(z)\left(\frac{1}{X_{0}\left(z_{\mathrm{CT}}\right)} \frac{w}{z-z_{\mathrm{CT}}}+\frac{1}{X_{0}\left(\bar{z}_{\mathrm{CT}}\right)} \frac{w}{z-\bar{z}_{\mathrm{CT}}}\right. \\
& \left.+\frac{1}{X_{0}\left(\bar{z}_{\mathrm{CT}}\right)} \frac{\bar{w}\left(z_{\mathrm{CT}}-\bar{z}_{\mathrm{CT}}\right)}{\left(z-\bar{z}_{\mathrm{CT}}\right)^{2}}+X_{0}\left(\bar{z}_{\mathrm{CT}}\right) \frac{\bar{w} \bar{z}_{\mathrm{CT}}\left(z_{\mathrm{CT}}-\bar{z}_{\mathrm{CT}}\right)}{z-\bar{z}_{\mathrm{CT}}}\right),
\end{aligned}
$$




$$
\begin{aligned}
& \Omega_{\perp}(z)= \frac{1}{2}\left(\frac{w}{z-z_{\mathrm{CT}}}-\frac{w}{z-\bar{z}_{\mathrm{CT}}}-\frac{\bar{w}\left(z_{\mathrm{CT}}-\bar{z}_{\mathrm{CT}}\right)}{\left(z-\bar{z}_{\mathrm{CT}}\right)^{2}}\right) \\
&-\frac{1}{2} X_{0}(z)\left(\frac{1}{X_{0}\left(z_{\mathrm{CT}}\right)} \frac{w}{z-z_{\mathrm{CT}}}+\frac{1}{X_{0}\left(\bar{z}_{\mathrm{CT}}\right)} \frac{w}{z-\bar{z}_{\mathrm{CT}}}\right. \\
&+\left.\frac{1}{X_{0}\left(\bar{z}_{\mathrm{CT}}\right)} \frac{\bar{w}\left(z_{\mathrm{CT}}-\bar{z}_{\mathrm{CT}}\right)}{\left(z-\bar{z}_{\mathrm{CT}}\right)^{2}}+X_{0}\left(\bar{z}_{\mathrm{CT}}\right) \frac{\bar{w}_{\mathrm{CT}}\left(z_{\mathrm{CT}}-\bar{z}_{\mathrm{CT}}\right)}{z-\bar{z}_{\mathrm{CT}}}\right), \\
& \Psi_{\perp}(z)=-\Phi_{\perp}(z)-z \Phi_{\perp}^{\prime}(z)-\bar{\Omega}_{\perp}(z) .
\end{aligned}
$$

Utilizing the Peach-Koehler formula [45], the self-image force $f_{\mathrm{I}}^{\mathrm{CT}}$ can be given as:

$$
\begin{aligned}
f_{\mathrm{I}}^{\mathrm{CT}} & =f_{x}-\mathrm{i} f_{y} \\
& =\left[\widehat{\tau}_{x y}\left(z_{\mathrm{CT}}\right) b_{x}+\widehat{\sigma}_{y}\left(z_{\mathrm{CT}}\right) b_{y}\right]+\mathrm{i}\left[\widehat{\sigma}_{x}\left(z_{\mathrm{CT}}\right) b_{x}+\widehat{\tau}_{x y}\left(z_{\mathrm{CT}}\right) b_{y}\right] \\
& =\frac{\mu b_{r}^{2}}{\pi(1+\kappa)}\left(\frac{\Phi_{\perp}^{*}\left(z_{\mathrm{CT}}\right)+\overline{\Phi_{\perp}^{*}\left(z_{\mathrm{CT}}\right)}}{w}+\frac{\bar{z}_{\mathrm{CT}} \Phi_{\perp}^{* \prime}\left(z_{\mathrm{CT}}\right)+\Psi_{\perp}^{*}\left(z_{\mathrm{CT}}\right)}{\bar{w}}\right),
\end{aligned}
$$

with $\kappa=3-4 v, \hat{\sigma}_{x}, \hat{\sigma}_{y}$ and $\hat{\tau}_{x y}$ are the components of the perturbation stress, and

$$
\begin{gathered}
\Phi_{\perp}^{*}\left(z_{\mathrm{CT}}\right)=\lim _{z \rightarrow z_{\mathrm{CT}}}\left(\Phi_{\perp}(z)-\Phi_{\perp}^{0}(z)\right), \\
\Phi_{\perp}^{* \prime}\left(z_{\mathrm{CT}}\right)=\lim _{z \rightarrow z_{\mathrm{CT}}} \frac{\mathrm{d}\left(\Phi_{\perp}(z)-\Phi_{\perp}^{0}(z)\right)}{\mathrm{d} z}, \\
\Psi_{\perp}^{*}\left(z_{\mathrm{CT}}\right)=\lim _{z \rightarrow z_{\mathrm{CT}}}\left(\Psi_{\perp}(z)-\Psi_{\perp}^{0}(z)\right) .
\end{gathered}
$$

Secondly, the force $f_{\Delta}^{\mathrm{CT}}$ caused by the GB sliding may be expressed as [45]:

$$
f_{\Delta}^{\mathrm{CT}}=\frac{\mu b_{r}^{2}}{\pi(1+\kappa)}\left(\frac{\Phi_{\Delta}\left(z_{\mathrm{CT}}\right)+\overline{\Phi_{\Delta}\left(z_{\mathrm{CT}}\right)}}{w}+\frac{\bar{z}_{\mathrm{CT}} \Phi_{\Delta}^{\prime}\left(z_{\mathrm{CT}}\right)+\Psi_{\Delta}\left(z_{\mathrm{CT}}\right)}{\bar{w}}\right) .
$$

Finally, the slip force $f_{\mathrm{A}}^{\mathrm{CT}}$ can be obtained as [44]:

$$
f_{\mathrm{A}}^{\mathrm{CT}}=b_{r} \tau_{r \theta}=\frac{b_{r} K_{\mathrm{I}}}{2 \sqrt{2 \pi r_{\mathrm{CT}}}} \sin \theta_{\mathrm{CT}} \cos \left(2 \theta_{\mathrm{e}}-\frac{3 \theta_{\mathrm{CT}}}{2}\right),
$$

where $K_{\mathrm{I}}$ is the stress intensity factor (SIF) produced by the applied mode I load.

Thus, for a crack tip dislocation, the emission force $f_{\text {emit }}^{\mathrm{CT}}$ can be expressed as: 


$$
\begin{aligned}
f_{\text {emit }}^{\mathrm{CT}} & =f_{x} \cos \theta_{\mathrm{e}}+f_{y} \sin \theta_{\mathrm{e}}+f_{\mathrm{A}}^{\mathrm{CT}} \\
& =\operatorname{Re}\left[f_{\mathrm{I}}^{\mathrm{CT}}+f_{\Delta}^{\mathrm{CT}}\right] \cos \theta_{\mathrm{e}}-\operatorname{Im}\left[f_{\mathrm{I}}^{\mathrm{CT}}+f_{\Delta}^{\mathrm{CT}}\right] \sin \theta_{\mathrm{e}}+f_{\mathrm{A}}^{\mathrm{CT}} .
\end{aligned}
$$

\subsection{Dislocation emission from $G B$}

For the first dislocaiton emitting from the GB, the emission force $f_{\text {emit }}^{\mathrm{GB}}$ is the sum of the self-image force $f_{\mathrm{I}}^{\mathrm{GB}}$, the force $f_{\Delta}^{\mathrm{GB}}$ induced by the GB sliding, the external force $f_{\mathrm{A}}^{\mathrm{GB}}$ and the GB transformation force $f_{\mathrm{GB}}$ produced by the GB transformation due to the dislocation emission .

By the same method used in Section 3.1, the forces $f_{\mathrm{I}}^{\mathrm{GB}}, f_{\Delta}^{\mathrm{GB}}$ and $f_{\mathrm{A}}^{\mathrm{GB}}$ acting on the GB dislocation located at the centre of the wedge dsiclination dipole $z_{0}=r_{0} \mathrm{e}^{\mathrm{i} \theta_{0}}$ with Burgers vector $b_{r} \mathrm{e}^{\mathrm{i} \theta_{G B}}$ can be derived. Referring to the work [46], the GB transformation force $f_{\mathrm{GB}}$ can be given as:

$$
f_{\mathrm{GB}}=-\frac{2 \Delta E_{\mathrm{GB}}}{\pi r_{\mathrm{GB}}},
$$

where $\Delta E_{\mathrm{GB}}$ is the energy change of the GB due to the dislocation emission and $r_{\mathrm{GB}}$ denotes the cutoff distance corresponding to the magnitude of the GB dislocation core size.

For a GB dislocation, the emission force $f_{\text {emit }}^{\mathrm{GB}}$ can be derived as

$$
\begin{aligned}
f_{\text {emit }}^{\mathrm{GB}} & =f_{x} \cos \theta_{\mathrm{GB}}+f_{y} \sin \theta_{\mathrm{GB}}+f_{\mathrm{A}}^{\mathrm{GB}}+f_{\mathrm{GB}} \\
& =\operatorname{Re}\left[f_{\mathrm{I}}^{\mathrm{GB}}+f_{\Delta}^{\mathrm{GB}}\right] \cos \theta_{\mathrm{GB}}-\operatorname{Im}\left[f_{\mathrm{I}}^{\mathrm{GB}}+f_{\Delta}^{\mathrm{GB}}\right] \sin \theta_{\mathrm{GB}}+f_{\mathrm{A}}^{\mathrm{GB}}+f_{\mathrm{GB}}
\end{aligned}
$$

\section{Critical stress intensity factors}

Following the literature [47], the criterion for dislocation emission is the total force on it is equal to zero. In addition, for dislocation emission form cracks, the distance from the dislocation to the crack surface should be larger than the dislocation core radius. For dislocation emission from the GB, only consider that from the wedge disclination dipole's 
center $z_{0}$. Therefore, let the emission force $f_{\mathrm{GB}}=0$ and $f_{\mathrm{CT}}=0$, the critical SIF (produced by the applied load) can be given.

For dislocation emission from the crack tip,

$$
K_{\mathrm{CT}}^{\mathrm{C}}=\frac{2 \sqrt{2 \pi r_{\mathrm{CT}}}}{b_{r} \sin \theta_{\mathrm{CT}} \cos \frac{\theta_{\mathrm{CT}}}{2}}\left(\operatorname{Im}\left[f_{\mathrm{I}}^{\mathrm{CT}}+f_{\Delta}^{\mathrm{CT}}\right] \sin \theta_{\mathrm{CT}}-\operatorname{Re}\left[f_{\mathrm{I}}^{\mathrm{CT}}+f_{\Delta}^{\mathrm{CT}}\right] \cos \theta_{\mathrm{CT}}\right)
$$

For dislocation emission from the GB,

$$
K_{\mathrm{GB}}^{\mathrm{C}}=\frac{2 \sqrt{2 \pi r_{0}}}{b_{r} \sin \theta_{0} \cos \left(2 \theta_{\mathrm{GB}}-\frac{3 \theta_{0}}{2}\right)}\left(\operatorname{Im}\left[f_{\mathrm{I}}^{\mathrm{GB}}+f_{\Delta}^{\mathrm{GB}}\right] \sin \theta_{\mathrm{GB}}-\operatorname{Re}\left[f_{\mathrm{I}}^{\mathrm{GB}}+f_{\Delta}^{\mathrm{GB}}\right] \cos \theta_{\mathrm{GB}}-f_{\mathrm{GB}}\right)(20)
$$

In subsequent calculation, normalize the critical SIFs by $\mu \sqrt{b_{r}}, K_{\mathrm{CT}}^{0}=K_{\mathrm{CT}}^{\mathrm{C}} /\left(\mu \sqrt{b_{r}}\right)$ and $K_{\mathrm{GB}}^{0}=K_{\mathrm{GB}}^{\mathrm{C}} /\left(\mu \sqrt{b_{r}}\right)$. The parameter values of NC Ni, $\mu=73 \mathrm{GPa}$ and $v=0.31$, are used. Moreover, define the magnitude of dislocation's Burgers vector $b_{r}=0.25 \mathrm{~nm}$ and the core radius $r_{\mathrm{CT}}=b_{r} / 2$. The angles $\theta_{\mathrm{CT}}$ and $\theta_{\mathrm{GB}}$ are respectively the emission angles of dislocations emitted from cracks and GBs. For convenience, introduce the angle $\theta_{\mathrm{GB}}^{\prime}=\theta_{\mathrm{GB}}-\alpha+\pi / 2$, which denotes the angle between the $\mathrm{GB}$ and the emitted dislocation. Note that the energy change of the GB due to one dislocation emission is really small, so that the transformation force $f_{\mathrm{GB}}$ for the first dislocation emission can be negelcted. In addition, the distance from the crack tip to the disclination dipole center $r_{0}$ is arbitrary as shown in Fig. 1. Though the distance $r_{0}$ is not in direct proportion to the grain size $d$, the range of $r_{0}$ is limited in $0<r_{0} \leq d$. To derived the grain size-dependent criterion for the first dislocation emission, let $r_{0}=\phi d$, where $0<\phi \leq 1$.

The normalized critical SIFs $K_{\mathrm{GB}}^{0}$ and $K_{\mathrm{CT}}^{0}$ versus the grain size $d$ with different disclination strength $\omega$ are depicted in Fig. 2. With the increasing grain size, $K_{\mathrm{GB}}^{0}$ always 
increases, while $K_{\mathrm{CT}}^{0}$ decreases to constants monotonously. With the same strength of GB sliding, the normalized critical SIFs $K_{\mathrm{GB}}^{0}$ and $K_{\mathrm{CT}}^{0}$ have an intersection at the critical point $d_{0}$. When the grain size is smaller than the critical value $d_{0}$, the dislocation emission from GBs will be first activated during the deformation. Otherwise, the dislocation emission from cracks will be first activated. This result demonstrates that the main dislocation source may transform from GBs to crack tips with the increasing grain size.

This can also explain that GB sliding can toughen the NC materials even though it suppresses dislocation emission from cracks when their grain sizes are relative small, which is because the dislocation emission from GBs is activated. Therefore, the ductility of nanomaterials with different grain sizes can be enhanced through the cooperative dislocation emission from crack tips and GBs.

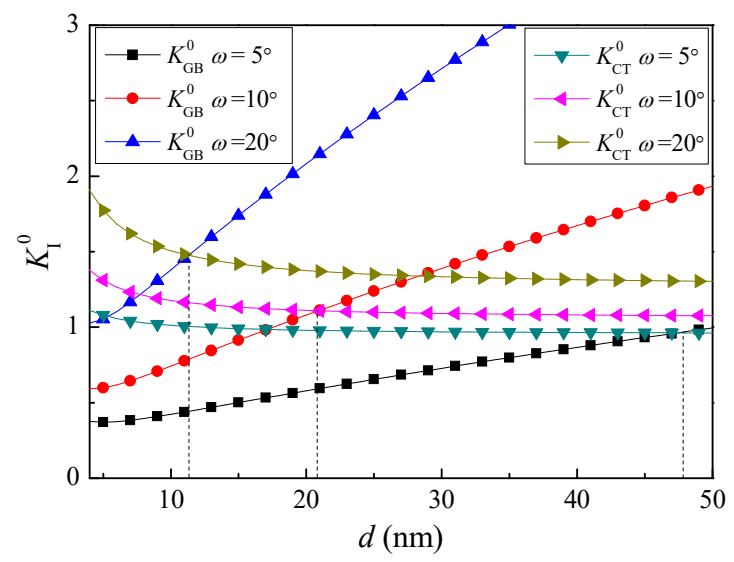

Figure 2. The normalized critical SIFs $K_{\mathrm{I}}^{0}$ versus $d$ with different $\omega$.

$$
\left(\phi=0.5, p=2 \mathrm{~nm}, \theta_{0}=45^{\circ}, \theta_{\mathrm{GB}}=45^{\circ}, \theta_{\mathrm{CT}}=30^{\circ}, \alpha=120^{\circ} \text { and } l=50 \mathrm{~nm}\right)
$$

Fig.3 describes the normalized critical SIFs $K_{\mathrm{GB}}^{0}$ and $K_{\mathrm{CT}}^{0}$ as functions of the GB's orientation angle $\alpha$ with different disclination strength $\omega$. As the reason that the force acting on the dislocaiton induced by the GB sliding is closely related to the orientation of the GB, the normalized critical SIFs can either be enhanced or weaken by the GB sliding, 
depending on the GB's orientation. For dislocation emission from cracks, $K_{\mathrm{CT}}^{0}$ decreases with the increasing disclination strength when the orientation of GB in the range $0^{\circ} \leq \alpha<60^{\circ}$ and $240^{\circ}<\alpha \leq 360^{\circ}$, which means that the dislocation emission is promoted by GB sliding. While when the GB's orientation in the range $60^{\circ}<\alpha<240^{\circ}, K_{\mathrm{CT}}^{0}$ increases with the increasing disclination strength indicating GB sliding can effectually suppress dislocation emission. At the same time, the $K_{\mathrm{CT}}^{0}$ first increases and then decreases with the increasing orientation of the GB. There is a critical orientation, where the normalized critical SIF gets the maximum, making the dislocation emission most difficult.

Following Huang and Li [48], the sign of the SIF is determined by the direction of the Burgers vector of the emerging dislocations. Therefore, the dislocation emission from GBs (at the center of wedge disclination dipole) can be suppressed by the GB sliding. The strength of GB sliding is stronger, the edge dislocation is more difficult to emit.

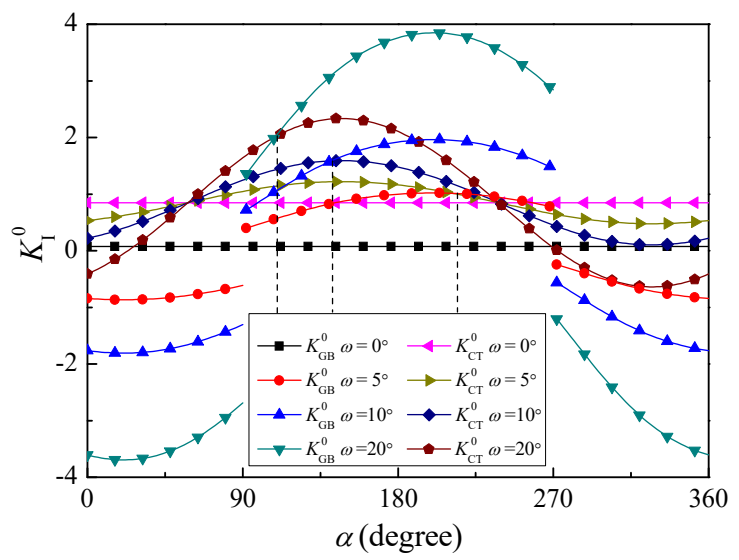

Figure 3. The normalized critical SIF $K_{\mathrm{I}}^{0}$ versus $\alpha$ with different $\omega$.

$$
\left(d=20 \mathrm{~nm}, \phi=0.5, p=5 \mathrm{~nm}, \theta_{0}=45^{\circ}, \theta_{\mathrm{GB}}=45^{\circ}, \theta_{\mathrm{CT}}=30^{\circ} \text { and } l=50 \mathrm{~nm}\right)
$$

What's more, when the GB sliding does not exist $\left(\omega=0^{\circ}\right)$, dislocation emission from GBs is easier than that from cracks. This is because the attraction associated with the self-image force $f_{\mathrm{I}}$ induced by cracks is relatively large if the dislocation is very close to the crack tip. 
Its magnitude will decrease with the increasing distance between the dislocation and crack tip. Then, for the same GB sliding strength, $K_{\mathrm{GB}}^{0}$ and $K_{\mathrm{CT}}^{0}$ intersect at the critical point $\alpha_{0}$. When the GB's orientation (in the range $90^{\circ} \leq \alpha \leq 270^{\circ}$ ) is smaller than the value $\alpha_{0}$, dislocation emission from GBs will be first activated during the deformation. Otherwise, dislocation emission from cracks will be first activated. In addition, the critical value $\alpha_{0}$ decreases with the increasing disclination strength.

The normalized critical SIFs $K_{\mathrm{GB}}^{0}$ and $K_{\mathrm{CT}}^{0}$ versus the disclination strength $\omega$ for different crack length $l$ are described in Fig. 4. In the situation where the orientation of the GB $\alpha=120^{\circ}$, the normalized critical SIFs $K_{\mathrm{GB}}^{0}$ and $K_{\mathrm{CT}}^{0}$ both increase monotonously with the increasing GB sliding strength. The normalized critical SIFs $K_{\mathrm{GB}}^{0}$ and $K_{\mathrm{CT}}^{0}$ for the same crack length intersect at the critical point $\omega_{0}$. When the disclination strength is smaller than $\omega_{0}$, dislocation emission from GBs will be first activated during the deformation. Otherwise, dislocation emission from cracks will be first activated. In addition, the critical value $\omega_{0}$ will increase with the increasing crack length.

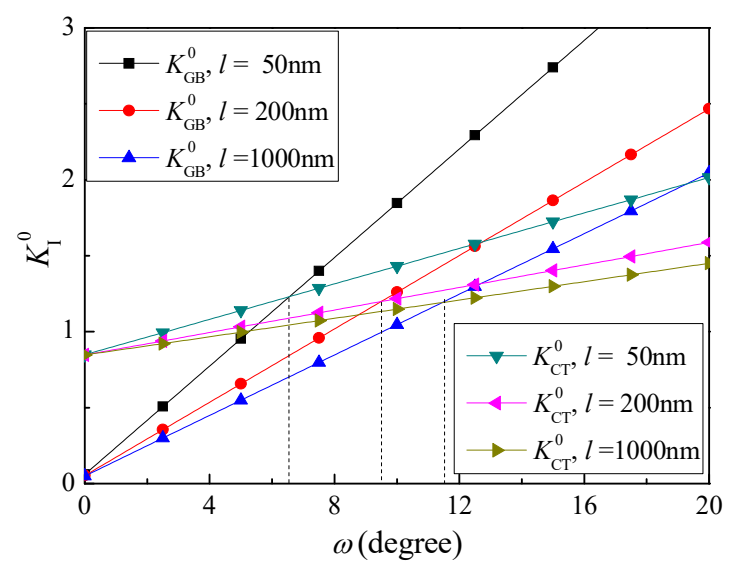

Figure 4. The normalized critical SIF $K_{\mathrm{I}}^{0}$ versus $\omega$ with different $l$.

$$
\left(d=40 \mathrm{~nm}, \phi=0.5, p=5 \mathrm{~nm}, \theta_{0}=45^{\circ}, \theta_{\mathrm{GB}}=45^{\circ}, \theta_{\mathrm{CT}}=30^{\circ} \text { and } \alpha=120^{\circ}\right)
$$




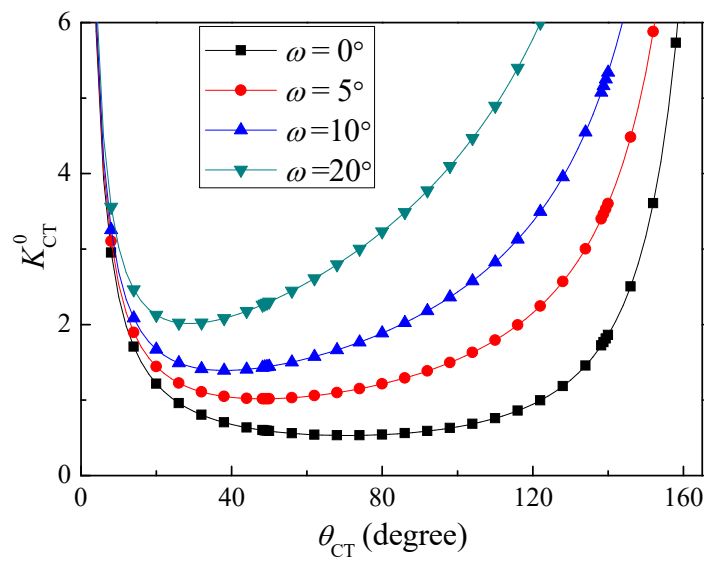

(a)

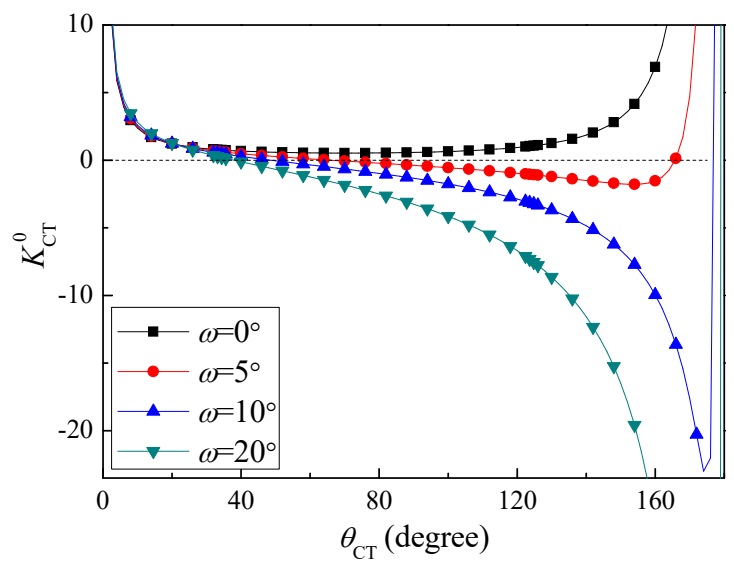

(b)

Figure 5. The normalized critical SIFs $K_{\mathrm{I}}^{0}$ versus $\theta_{\mathrm{CT}}$ with different $\omega$, (a) $\alpha=120^{\circ}$ and (b) $\alpha=45^{\circ} .\left(d=40 \mathrm{~nm}, \phi=0.5, p=5 \mathrm{~nm}, \quad \theta_{0}=45^{\circ}\right.$ and $\left.l=50 \mathrm{~nm}\right)$

Fig. 5 dispicts the normalized critical SIFs $K_{\mathrm{CT}}^{0}$ as functions of the dislocation emission angle $\theta_{\mathrm{Cт}}$ with different disclination strength $\omega$. When the orientation of the GB $\alpha=120^{\circ}$, the normalized critical SIF $K_{\mathrm{CT}}^{0}$ first diminishes from positive infinity to a minimum and then goes up with the increasing emission angle $\theta_{\mathrm{CT}}$ as shown in Fig. 5(a). The minimum persents the most probable angle $\theta_{\mathrm{CT}}$ for dislocation emission. What is more, the most probable emission angle decreases with the increasing disclination strength $\omega$. In Fig. 5(b), 
$K_{\mathrm{CT}}^{0}$ also first diminishes from positive infinity to a minimum and then goes up with the increasing emission angle $\theta_{\mathrm{CT}}$ in the situation where the GB's orientation $\alpha=45^{\circ}$. The difference is that the minimum will be negative when the disclination strength $\omega$ is strong enough, in which case there will be two most probable angles for dislocation emission from cracks.

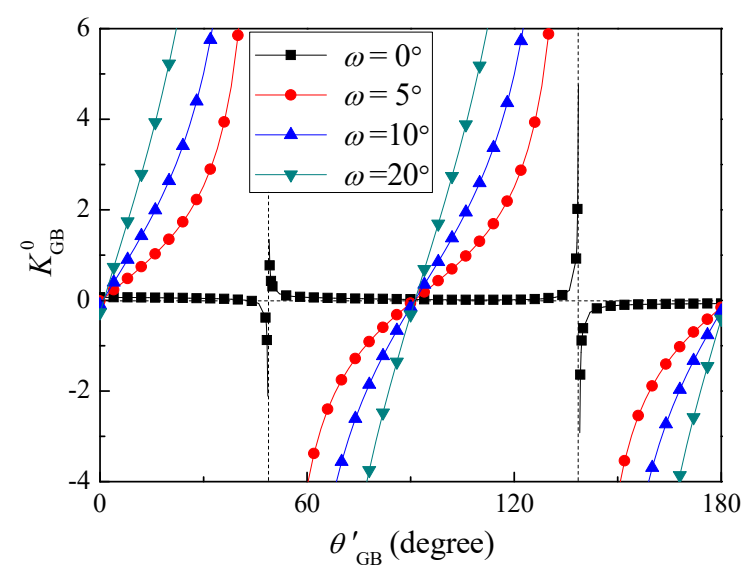

(a)

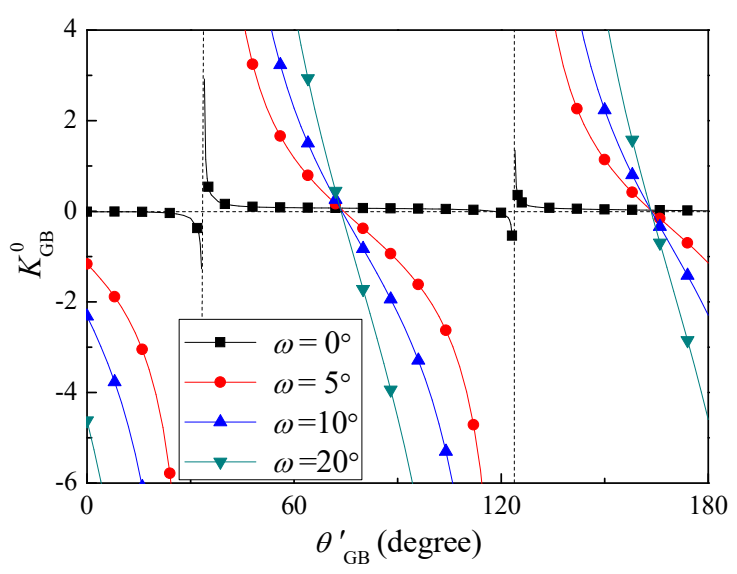

(b)

Figure 6. The normalized critical SIFs $K_{\mathrm{I}}^{0}$ versus $\theta_{\mathrm{GB}}^{\prime}$ with different $\omega$, (a) $\alpha=120^{\circ}$ and (b) $\alpha=45^{\circ} .\left(d=40 \mathrm{~nm}, \phi=0.5, p=5 \mathrm{~nm}, \theta_{0}=45^{\circ}\right.$ and $\left.l=50 \mathrm{~nm}\right)$

The variation of the normalized critical SIF $K_{\mathrm{GB}}^{0}$ with different disclination strength $\omega$ on the angle $\theta_{\mathrm{GB}}^{\prime}$ (angle between the $\mathrm{GB}$ and the emitted dislocation from the $\mathrm{GB}$ ) is 
described in Fig. 6. It can be seen that the normalized critical SIFs have totally different variation tendencies compared to the situation where the GB sliding vanished $\left(\omega=0^{\circ}\right)$. When GB sliding exists and the orientation of the GB $\alpha=120^{\circ}$, the normalized critical SIF changes periodically with the angle $\theta_{\mathrm{GB}}^{\prime}$ and the period is $\pi / 2$. There are points, $\theta_{\mathrm{GB}}^{\prime} \approx 0^{\circ}$ and $90^{\circ}$, where the normalized critical SIF $K_{\mathrm{GB}}^{0}$ equals to zero. This means that the dislocations are more inclined to emit along the GB or perpendicular to the GB under this situation. When the orientation of the GB $\alpha=45^{\circ}$, there are also two most probable angles $\left(\theta_{\mathrm{GB}}^{\prime} \approx 73^{\circ}\right.$ and $163^{\circ}$ ) for dislocation emission from the GB.

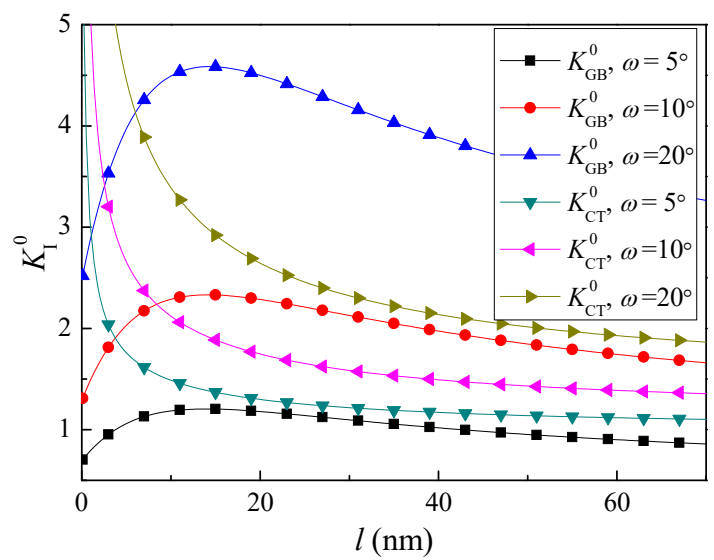

Figure 7. The normalized critical SIFs $K_{\mathrm{I}}^{0}$ versus $l$ with different $\omega$.

$$
\left(d=40 \mathrm{~nm}, \phi=0.5, p=5 \mathrm{~nm}, \theta_{0}=45^{\circ}, \theta_{\mathrm{GB}}=45^{\circ}, \theta_{\mathrm{CT}}=30^{\circ} \text { and } \alpha=120^{\circ}\right)
$$

The normalized critical SIFs $K_{\mathrm{GB}}^{0}$ and $K_{\mathrm{CT}}^{0}$ versus the crack length $l$ with different disclination strength $\omega$ are depicted in Fig. 7. In the situation where the GB's orientation $\alpha=120^{\circ}, K_{\mathrm{GB}}^{0}$ firstly goes up to a maximum and then diminishes to constant with the increasing crack length, while the normalized critical SIF $K_{\mathrm{CT}}^{0}$ decreases monotonously to constants. Therefore, there is a critical crack length making dislocation emission from GBs most difficult. Also, dislocations are easier to emit from the tip of longer cracks. 
In addition, when the disclination strength $\omega=5^{\circ}, K_{\mathrm{GB}}^{0}$ is always smaller than $K_{\mathrm{CT}}^{0}$, which means dislocation emission from GBs will be first activated during the deformation. However, when the strength of GB sliding is strong enough, the normalized critical SIFs $K_{\mathrm{GB}}^{0}$ and $K_{\mathrm{CT}}^{0}$ with the same disclination strength have an intersection at the crack length $l_{0}$. This indicates that the dislocation emission from GBs will be first activated if the crack length is smaller than $l_{0}$. Otherwise, the dislocation emission from cracks will be first activated.

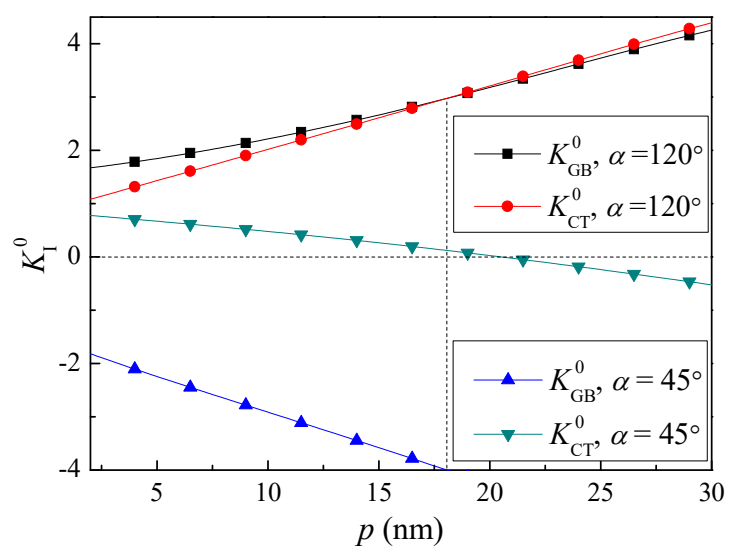

Figure 8. The normalized critical SIFs $K_{\mathrm{I}}^{0}$ versus $p$ with different $\omega$.

$$
\left(\omega=10^{\circ}, d=40 \mathrm{~nm}, \phi=0.5, \theta_{0}=45^{\circ}, \theta_{\mathrm{GB}}=45^{\circ}, \theta_{\mathrm{CT}}=30^{\circ} \text { and } l=50 \mathrm{~nm}\right)
$$

Fig. 8 shows the normalized critical SIFs $K_{\mathrm{GB}}^{0}$ and $K_{\mathrm{CT}}^{0}$ versus the arm length $p$ (the distance of GB sliding) with different disclination strength $\omega$. When the orientation of the GB $\alpha=45^{\circ}$, the direction of the Burgers vector of the dislocation emitted from the crack tip will chang with the increasing GB sliding distance. Meanwhile, the dislocation emission from cracks is always easier than that from GBs. $K_{\mathrm{GB}}^{0}$ and $K_{\mathrm{CT}}^{0}$ both increase monotonously with the increasing arm length $p$ when the orientation of the GB $\alpha=120^{\circ}$ and there is an intersection at the critical point $p_{0}$. This means that the critical SIFs $K_{\mathrm{GB}}^{0}$ and $K_{\mathrm{CT}}^{0}$ are enhanced by the increasing GB sliding distance. When the GB sliding distance is smaller than 
$p_{0}$, the dislocation emission from cracks will be first activated during the deformation. Otherwise, the dislocation emission from GBs will be first activated.

\section{Conclusion}

In summary, the cooperative mechanism between the dislocation emission from cracks and GBs driven by GB sliding in deformed NC materials is investigated. Within the model, the high local stress concentration nearby the crack tip can initiate GB sliding which creates a disclination dipole at the triple junction of GBs. Then, it can serve as a stress source providing for the dislocation emitting from GBs and influencing the dislocation emission from cracks. The grain size-dependent criterions for the dislocation emission from the crack tip and the GB are respectively derived. Influences of GB sliding and grain size on the cooperative mechanism is discussed.

The results indicate that the grain size significantly impact the dislocation emission. For small grain sizes, dislocation emission from GBs is activated ahead of that from cracks. This can explain that GB sliding can toughen the NC materials even though it suppresses dislocation emission from cracks when grain sizes are relative small, which is because the dislocation emission from GBs is activated. With the increasing grain size, the main dislocation source may transform from GBs to crack tips due to GB sliding. Therefore, the ductility of nanomaterials with different grain sizes can be enhanced through the cooperative dislocation emission from cracks and GBs.

Dislocation emission can either be promoted or suppressed by the GB sliding, depending on the orientation of the GB. For some certain parameters, there may be critical grain size, GB's orientation and disclination strength, which will change the priority mechanism of dislocation emission from cracks or from GBs.

\section{Acknowledgements}


The authors would like to deeply appreciate the support from the National Natural Science Foundation of China (11572118, 11602080, 11772122 and 51778551), Hunan Provincial Natural Science Foundation of China (2018JJ3026) and the Fundamental Research Funds for the Central Universities.

\section{References}

[1] M. Dao, L. Lu, R. Asaro, J. Dehosson, E. Ma, Toward a quantitative understanding of mechanical behavior of nanocrystalline metals, Acta Materialia, 55 (2007) 4041-4065.

[2] C.C. Koch, Structural nanocrystalline materials: an overview, Journal of Materials Science, 42 (2007) 1403-1414.

[3] I.A. Ovid'ko, R.Z. Valiev, Y.T. Zhu, Review on superior strength and enhanced ductility of metallic nanomaterials, Progress in Materials Science, 94 (2018) 462-540.

[4] K. Zhou, A. Nazarov, M.S. Wu, Continuum and atomistic studies of a disclinated crack in a bicrystalline nanowire, Physical Review B, 73 (2006) 045410.

[5] K. Zhou, M.S. Wu, A. Nazarov, Relaxation of a disclinated tricrystalline nanowire, Acta Materialia, 56 (2008) 5828-5836.

[6] I.A. Ovid'ko, A.G. Sheinerman, Grain boundary sliding, triple junction disclinations and strain hardening in ultrafine-grained and nanocrystalline metals, International Journal of Plasticity, 96 (2017) 227-241.

[7] V. Borovikov, M.I. Mendelev, A.H. King, Effects of solutes on dislocation nucleation from grain boundaries, International Journal of Plasticity, 90 (2017) 146-155.

[8] M. Meraj, C. Deng, S. Pal, Stress-induced solid-state amorphization of nanocrystalline Ni and NiZr investigated by atomistic simulations, Journal of Applied Physics, 123 (2018) 044306.

[9] K.N. Mikaelyan, M.Y. Gutkin, E.N. Borodin, A.E. Romanov, Dislocation emission from the edge of a misfitting nanowire embedded in a free-standing nanolayer, International Journal of Solids and Structures, 161 (2019) 127-135.

[10] F. Zhang, Y. Liu, J. Zhou, The crack nucleation in hierarchically nanotwinned metals, Engineering Fracture Mechanics, 201 (2018) 29-35. 
[11] R.B. Figueiredo, M. Kawasaki, T. Langdon, The mechanical properties of ultrafine-grained metals at elevated temperatures, Rev. Adv. Mater. Sci, 19 (2009) 1-12.

[12] Y. Pei, D. Galvan, J.T.M. De Hosson, Nanostructure and properties of TiC/aC: H composite coatings, Acta materialia, 53 (2005) 4505-4521.

[13] A. Sergueeva, N. Mara, N. Krasilnikov, R. Valiev, A. Mukherjee, Cooperative grain boundary sliding in nanocrystalline materials, Philosophical Magazine, 86 (2006) 5797-5804.

[14] P. Kumar, M. Kawasaki, T.G. Langdon, Review: Overcoming the paradox of strength and ductility in ultrafine-grained materials at low temperatures, Journal of Materials Science, $51(2015) 7-18$.

[15] R.Z. Valiev, Y.T. Zhu, Recent Findings in Superior Strength and Ductility of Ultrafine-Grained Materials, Transactions of the Materials Research Society of Japan, 40 (2015) 309-318.

[16] A.G. Sheinerman, N.F. Morozov, M.Y. Gutkin, Effect of grain boundary sliding on fracture toughness of ceramic_graphene composites, Mechanics of Materials, 137 (2019) 103126.

[17] C.H. Liu, W.J. Lu, G.J. Weng, J.J. Li, A cooperative nano-grain rotation and grain-boundary migration mechanism for enhanced dislocation emission and tensile ductility in nanocrystalline materials, Materials Science and Engineering: A, 756 (2019) 284-290.

[18] T.W. He, W.S. Xiao, Q.H. Fang, H.P. Zhu, M.L. Feng, Influence of grain boundary sliding near a nanovoid on crack growth in deformed nanocrystalline materials, International Journal of Mechanical Sciences, 144 (2018) 842-848.

[19] C.H. Liu, W.J. Lu, S.H. Chen, J.J. Li, Toughening of nanocrystalline materials by nanograin rotation, Materials Today Communications, 19 (2019) 297-299.

[20] F.S. Tan, Q.H. Fang, J. Li, H. Feng, Enhanced nanotwinning by special grain growth in nanocrystalline materials, Journal of Materials Science, 55 (2020) 3618-3628.

[21] K. Zhou, M.S. Wu, Stress field of a disclination dipole in hep bicrystal with imperfect interface, International Journal of Engineering Science, 48 (2010) 237-252.

[22] M. Yu, Y. Yang, X. Peng, P.H. Wen, Effect of nanotwin and dislocation pileup at twin boundary on dislocation emission from a semi-elliptical blunt crack tip in nanocrystalline materials, Engineering Fracture Mechanics, 202 (2018) 288-296. 
[23] J. Weissmüller, J. Markmann, Deforming nanocrystalline metals: New insights, new puzzles, Advanced Engineering Materials, 7 (2005) 202-207.

[24] S. Liang, Y.X. Zhu, M.S. Huang, Z.H. Li, Simulation on crack propagation vs. crack-tip dislocation emission by XFEM-based DDD scheme, International Journal of Plasticity, 114 (2019) 87-105.

[25] Y. Li, X. Ren, J. He, Z. Zhang, Constraint effect on the brittle-to-ductile transition of single-crystal iron induced by dislocation mobility, International Journal of Mechanical Sciences, 149 (2018) 212-223.

[26] S. Taketomi, R. Matsumoto, N. Miyazaki, Atomistic study of the effect of hydrogen on dislocation emission from a mode II crack tip in alpha iron, International Journal of Mechanical Sciences, 52 (2010) 334-338.

[27] S. Liang, Y. Zhu, M. Huang, Z. Li, Studying dislocation-induced shielding effect on the crack-tip in polycrystal by discrete dislocation dynamics, International Journal of Solids and Structures, 156-157 (2019) 148-162.

[28] X. Li, X. Jiang, Effects of dislocation pile-up and nanocracks on the main crack propagation in crystalline metals under uniaxial tensile load, Engineering Fracture Mechanics, 212 (2019) 258-268.

[29] X. Li, X. Jiang, Theoretical analyses of nanocrack nucleation near the main crack tip in nano and micro crystalline materials, Engineering Fracture Mechanics, 221 (2019) 106672.

[30] K. Kumar, S. Suresh, M. Chisholm, J. Horton, P. Wang, Deformation of electrodeposited nanocrystalline nickel, Acta Materialia, 51 (2003) 387-405.

[31] V. Borovikov, M.I. Mendelev, A.H. King, Effects of Ag and Zr solutes on dislocation emission from $511(332)[110]$ symmetric tilt grain boundaries in $\mathrm{Cu}$ : Bigger is not always better, International Journal of Plasticity, 109 (2018) 79-87.

[32] V. Dupont, F. Sansoz, Quasicontinuum study of incipient plasticity under nanoscale contact in nanocrystalline aluminum, Acta Materialia, 56 (2008) 6013-6026.

[33] L. Wang, Z. Zhang, E. Ma, X. Han, Transmission electron microscopy observations of dislocation annihilation and storage in nanograins, Applied Physics Letters, 98 (2011) 051905.

[34] S. Bobylev, M.Y. Gutkin, I. Ovid'ko, Transformations of grain boundaries in deformed nanocrystalline materials, Acta Materialia, 52 (2004) 3793-3805. 
[35] H. Van Swygenhoven, P. Derlet, A. Frøseth, Nucleation and propagation of dislocations in nanocrystalline fcc metals, Acta Materialia, 54 (2006) 1975-1983.

[36] I.A. Ovid'ko, N.V. Skiba, Enhanced dislocation emission from grain boundaries in nanocrystalline materials, Scripta Materialia, 67 (2012) 13-16.

[37] S. Bobylev, N. Morozov, I. Ovid'ko, Cooperative grain boundary sliding and migration process in nanocrystalline solids, Physical review letters, 105 (2010) 055504.

[38] H. Feng, Q.H. Fang, L.C. Zhang, Y.W. Liu, Special rotational deformation and grain size effect on fracture toughness of nanocrystalline materials, International Journal of Plasticity, 42 (2013) 50-64.

[39] A.E. Romanov, A.L. Kolesnikova, Application of disclination concept to solid structures, Progress in materials science, 54 (2009) 740-769.

[40] A. Romanov, V. Vladimirov, Disclinations in solids, Physica status solidi (a), 78 (1983) 11-34.

[41] N. Muskhelishvili, Some basic problems of the mathematical theory of elasticity, in, Nauka, Moscow, 1966.

[42] Q.H. Fang, Y.W. Liu, Elastic interaction between wedge disclination dipole and internal crack, Applied Mathematics and Mechanics, 27 (2006) 1239-1247.

[43] Y.W. Liu, Q.H. Fang, C.P. Jiang, Interaction of a wedge disclination dipole with circular inclusion, Phys. Status Solidi A, 203 (2006) 443.

[44] H.B. Zhao, H. Feng, F. Liu, Y. Liu, P.H. Wen, Effect of nanoscale twin and dislocation pileup at twin boundary on crack blunting in nanocrystalline materials, Acta Mechanica, 228 (2017) 3483-3495.

[45] J. Hirth, J. Lothe, Theory of Dislocations, 2nd, Ed.: John Willey \& Sons, (1982).

[46] T. Shimokawa, M. Tsuboi, Atomic-scale intergranular crack-tip plasticity in tilt grain boundaries acting as an effective dislocation source, Acta Materialia, 87 (2015) 233-247.

[47] J.R. Rice, R. Thomson, Ductile versus brittle behaviour of crystals, Philosophical magazine, 29 (1974) 73-97.

[48] M.X. Huang, Z.H. Li, Dislocation emission criterion from a blunt crack tip, Journal of the Mechanics and Physics of Solids, 52 (2004) 1991-2003. 